\title{
Early and midterm results of thoracic endovascular aortic repair of chronic type B aortic dissection
}

\author{
Shang Dong Xu, MD, ${ }^{\mathrm{a}}$ Fang Jiong Huang, MD, ${ }^{\mathrm{a}}$ Jin Fei Yang, MD, ${ }^{\mathrm{a}}$ Zhi Zhong Li, MD, ${ }^{\mathrm{b}}$ Sheng Yang, MD, \\ Jia Hui Du, MD, ${ }^{c}$ and Zhao Guang Zhang, MD $^{\mathrm{a}}$
}

Objective: The optimal treatment for chronic type B dissection remains controversial. This study reports early and midterm results of thoracic endovascular aortic repair for chronic type B aortic dissection.

\begin{abstract}
Methods: From June 2001 to September 2007, a total of 84 patients with chronic type B aortic dissection underwent thoracic endovascular aortic repair. The time between onset of dissection and thoracic endovascular aortic repair was $13.9 \pm 22.0$ months (range, $1-120$ months). All patients were followed up from 6 to 86 months (mean, $33.2 \pm 19.2$ months).

Results: The entry tear was completely sealed in 77 cases $(91.7 \%)$ during thoracic endovascular aortic repair. The incidence of incomplete seal was $8.3 \%$. The 1-month mortality was $1.2 \%$. One patient had retrograde type A dissection 1 month after the operation. Four patients underwent a second thoracic endovascular aortic repair during follow-up, for endoleak in 3 patients and for newly formed intimal tear in 1 patient. Seven patients $(8.3 \%)$ died during follow-up. Three died of rupture of the thoracic aorta because of endoleak. The Kaplan-Meier actuarial survival curve showed a 5-year survival of $84.4 \%$. At 5 years, $75.2 \%$ of patients were alive with neither endoleak nor reintervention.
\end{abstract}

Conclusions: Early and midterm results show that thoracic endovascular aortic repair was effective in the treatment of chronic type B aortic dissection. Endoleak was the main cause of death during follow-up. With increased surgical experience and refinement of the stent graft, results are likely to improve in the future. (J Thorac Cardiovasc Surg 2010;139:1548-53)

Since the introduction of endovascular repair of aortic disease by Volodos and colleagues ${ }^{1}$ and Dake and associates $^{2}$ thoracic endovascular aortic repair (TEVAR) has been increasingly used to treat type B aortic dissection. Clinical trials have reported the efficacy of TEVAR for treatment of type B dissection. ${ }^{3-5}$ Our own experience with TEVAR in acute type $\mathrm{B}$ aortic dissection has been reported previously. ${ }^{6}$ Chronic type B dissection differs from acute type B dissection in many aspects. The outcome of TEVAR in treatment of chronic type B dissection warrants a separate study, because there is little information focusing on TEVAR for chronic type B dissection. Between June 2001 and September 2007 , a total of 84 patients with chronic type B aortic dissection underwent TEVAR at our center. The early and midterm results are presented here.

From the Divisions of Cardiac Surgery, ${ }^{\mathrm{a}}$ Cardiology, ${ }^{\mathrm{b}}$ and Radiology, ${ }^{\mathrm{c}}$ Beijing Institute of Heart, Lung \& Vascular Diseases, Capital Medical University, Beijing Anzhen Hospital, Beijing, China.

Disclosures: None.

Received for publication March 28, 2009; revisions received July 30, 2009; accepted for publication Aug 23, 2009; available ahead of print Nov 12, 2009.

Address for reprints: Shang Dong Xu, MD, Beijing Anzhen Hospital, Cardiovascular Division, An Zhen Li, Chao Yang Qu, Beijing 100029, China (E-mail: xushangdong@vip.sina.com).

$0022-5223 / \$ 36.00$

Copyright (c) 2010 by The American Association for Thoracic Surgery doi: $10.1016 /$ j.jtcvs.2009.08.051

\section{MATERIALS AND METHODS \\ Patients}

Eighty-four patients with chronic type B aortic dissection underwent TEVAR between June 2001 and September 2007. The study included 69 male and 15 female patients, mean age $53.3 \pm 11.6$ years (range, $32-78$ years). Inclusion criteria were as follows: (1) type B aortic dissection confirmed by magnetic resonance angiography or computed tomographic angiography (CTA) with patent false lumen and no intramural hematoma, (2) time between onset of dissection and admission longer than 1 month, (3) arch diameter (landing zone) less than $38 \mathrm{~mm}$, (4) adequate access route, and (5) no aberrant right subclavian artery. From January 2005 onward, 2 more inclusion criteria were added: (1) distance between entry tear and opening of left subclavian artery (LSCA) more than $10 \mathrm{~mm}$, (2) no Marfan syndrome or any suspected connective tissue diseases. Informed consent was obtained from each patient. The study was approved by the ethics committee of our center. The patients' clinical data are listed in Table 1. All TEVAR procedures were elective, except for 3 emergency cases with contained rupture. The landing zone diameter (healthy aortic segment proximal to the dissection, usually the aortic arch where LSCA branches off) was measured with magnetic resonance angiography or CTA. Patients who were not suitable candidates for TEVAR underwent surgical treatment. During the same period, 6 patients with chronic type B dissection underwent surgery.

\section{Stent Grafts}

Five stent graft systems were used: Talent (Medtronic, Inc, Minneapolis, Minn) in 33 cases, EndoFit (Lemaitre Vascular, Inc, Burlington, Mass) in 1 case, Hercules (MicroPort, Shanghai, China) in 27 cases, Vasoflow (Vascore, Suzhou, China) in 3 patients, and Grikin (Grikin Advanced Materials Co, Ltd, Beijing, China) in 20 cases. All stent grafts had a metal skeleton covered by polyester or polytetrafluoroethylene. The Grikin stent graft does not have a longitudinal connecting bar, and the whole piece is tapered. 


\section{Abbreviations and Acronyms}

CTA = computed tomographic angiography

LSCA $=$ left subclavian artery

TEVAR $=$ thoracic endovascular aortic repair

\section{TEVAR Procedure}

TEVAR was performed in a catheter operating theater by a team of cardiovascular surgeons (S.D.X., F.J.H., J.F.Y., S.Y.), interventional cardiologists (Z.Z.L.), radiologists (J.H.D.), and an anesthetist. General anesthesia was used in 46 cases. Epidural anesthesia was used in 23 cases, and local anesthesia in 15 cases. The TEVAR procedure has been described in detail previously. ${ }^{6}$ In brief, the common femoral artery was exposed by surgical dissection unilaterally. With the calibrated pigtail catheter, the distance between entry tear and the opening of the LSCA was measured. A stent graft with a larger diameter (usually $10 \%-20 \%$ larger) was chosen. The delivery sheath was introduced over a stiff guide wire to the appropriate position. After the systolic blood pressure had been lowered to less than $100 \mathrm{~mm} \mathrm{Hg}$ and the heart rate lowered to less than 90 beats/min, the stent graft was deployed under fluoroscopy. Figure 1 shows the digital subtraction angiogram of a patient with type B dissection before and after stent graft implantation. If the entry tear was incompletely sealed, a cuff was added. Any reentry found in the thoracic aorta was also sealed by adding a cuff or cuffs. Three stent grafts were implanted in 1 patient, 2 stent grafts in 7 patients, and 1 stent graft in the remaining 76 patients. The opening of the LSCA was totally covered by the stent graft in 4 patients.

\section{Postoperative Care}

All patients were transferred to the intensive care unit after surgery. The systolic blood pressure was lowered to less than $120 \mathrm{~mm} \mathrm{Hg}$ by intravenous drugs. Ventilatory assistance was withdrawn after each patient became fully awake. Oral medication was given to control blood pressure. An intravenous antibiotic was used for 3 postoperative days. No anticoagulants were used. Most patients were ambulatory on postoperative day 1 .

\section{Follow-up}

CTA was conducted before discharge and at least once a year after surgery (Figure 2).

\section{Statistical Analysis \\ Clinical data were collected in a database. All data are expressed as mean $\pm \mathrm{SD}$.}

\section{RESULTS}

\section{Early Results}

The time between onset of dissection and TEVAR was $13.9 \pm 22.0$ months (range, 1-120 months). Early results are listed in Table 2. In 7 patients, the entry tear was incompletely sealed. The incidence of endoleak was $8.3 \%$. In other patients, the entry tears were sealed completely by the stent graft. One patient died of rupture of the thoracic aorta on postoperative day 1, 8 hours after TEVAR. Just before death, the patient had nausea and vomited. The blood pressure increased to $220 / 140 \mathrm{~mm} \mathrm{Hg}$ and dropped suddenly and dramatically, even before we tried to titrate up the nitroprusside. Thoracentesis confirmed left hemothorax. Sudden rupture of the descending thoracic aorta was considered the
TABLE 1. Patients' clinical data $(\mathbf{N}=\mathbf{8 4})$

\begin{tabular}{lc}
\hline Sex (no.) & $69(82.1 \%)$ \\
Male & $15(17.9 \%)$ \\
Female & \\
Age (y) & $32-78$ \\
$\quad$ Range & $53.3 \pm 11.6$ \\
Mean \pm SD & \\
Symptoms (no.) & $61(72.6 \%)$ \\
Back pain & $3(3.6 \%)$ \\
Shock & \\
Cause of aortic dissection (no.) & $67(79.8 \%)$ \\
Hypertension & $10(11.9 \%)$ \\
Atherosclerosis & $1(1.2 \%)$ \\
Marfan syndrome & $6(7.1 \%)$ \\
Unknown & $26(31.0 \%)$ \\
Smoking (no.) & $9(10.7 \%)$ \\
Diabetes mellitus (no.) & $3(3.6 \%)$ \\
Contained rupture (no.) & \\
Maximum descending aortic dimension (mm) & $45-80$ \\
Range & $58.1 \pm 9.8$ \\
Mean \pm SD & \\
Time from dissection to treatment (mo) & $1-120$ \\
Range & $13.9 \pm 22.0$ \\
Mean \pm SD & \\
\hline
\end{tabular}

cause of death. One-month mortality was $1.2 \%$. Three patients with contained rupture recovered smoothly. Two of them underwent minithoracotomy to remove clots in the chest cavity 1 week after the procedure. One of them underwent thoracentesis to drain bloody fluid out of the chest postoperatively. No neurologic defect followed the procedure. LSCA was totally occluded in 4 patients. None of these patients had left upper extremity ischemia or reduced cerebral blood supply. CTA before discharge showed that the false lumen in the descending thoracic aorta was partially thrombosed in the 7 patients with endoleaks. In the other patients, the false lumen in the descending thoracic aorta was totally thrombosed.

\section{Midterm Results}

All patients were followed up from 6 to 86 months (mean, $33.2 \pm 19.2$ months). One patient had a retrograde type A dissection 1 month after TEVAR. Emergency replacement of the ascending aorta and arch was conducted.

Three of the 7 patients with endoleaks underwent a second TEVAR procedure. A cuff was added at the proximal end of the first stent graft, and the entry tear was totally sealed. The other 4 patients with endoleaks all had entry tears immediately distal to the opening of the LSCA. Although the opening of the LSCA was used as the landing zone and was partially or totally covered in these 4 patients, a small amount of blood still entered the false lumen through the entry tear. These patients refused open surgery. Three died of rupture of the descending thoracic aorta during follow-up. One remains alive under close supervision. 

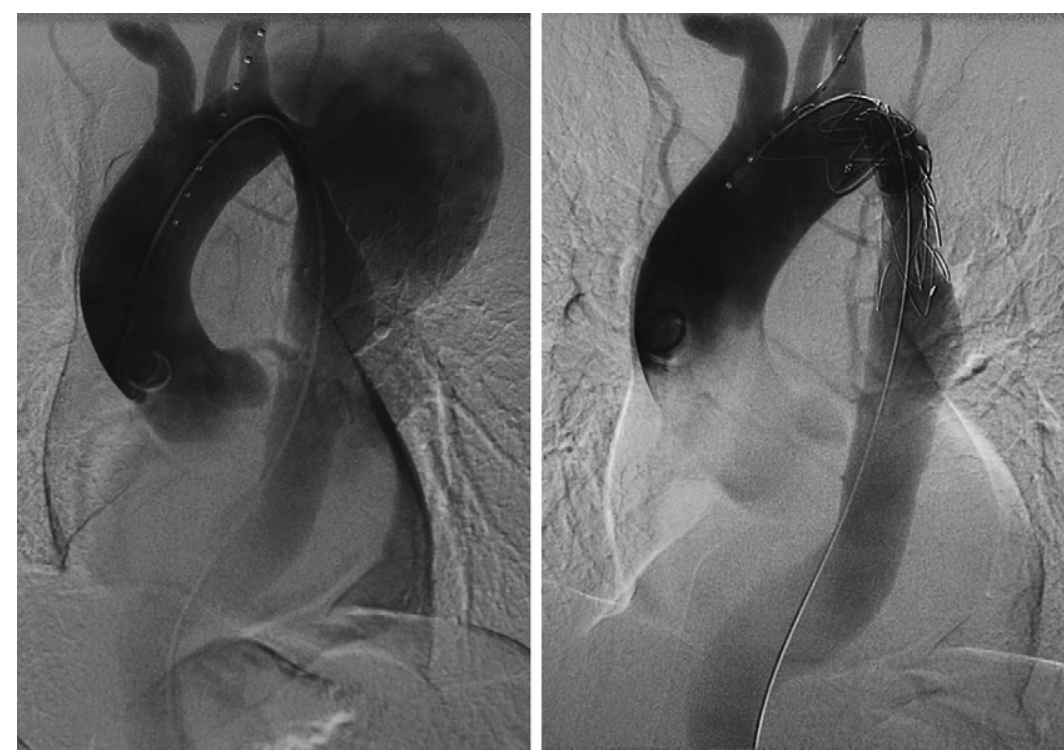

FIGURE 1. Digital subtraction angiogram of patient with type B dissection during thoracic endovascular aortic repair before (A) and after (B) stent graft implantation.

One patient underwent a second TEVAR procedure for a newly formed intimal tear caused by the distal end of the stent graft (Figure 3).

\section{Mortality}

In total, 7 patients died within the follow-up period. Mortality during follow-up was $8.3 \%$. Three patients with endoleaks died of rupture of the thoracic aorta. One patient with Marfan syndrome died of rupture of the abdominal aorta caused by continuous dilation of the abdominal aorta.
Two patients died of diseases unrelated to aortic dissection, 1 of cancer and the other of complications of diabetes. One patient died of an unknown cause. The Kaplan-Meier actuarial survival curve showed a 5-year survival of $84.4 \%$ (Figure 4 ). At 5 years, $75.2 \%$ of patients were alive with neither endoleak nor reintervention (Figure 5).

\section{DISCUSSION}

There is still controversy regarding the optimal treatment for type B aortic dissection. Traditional treatment includes
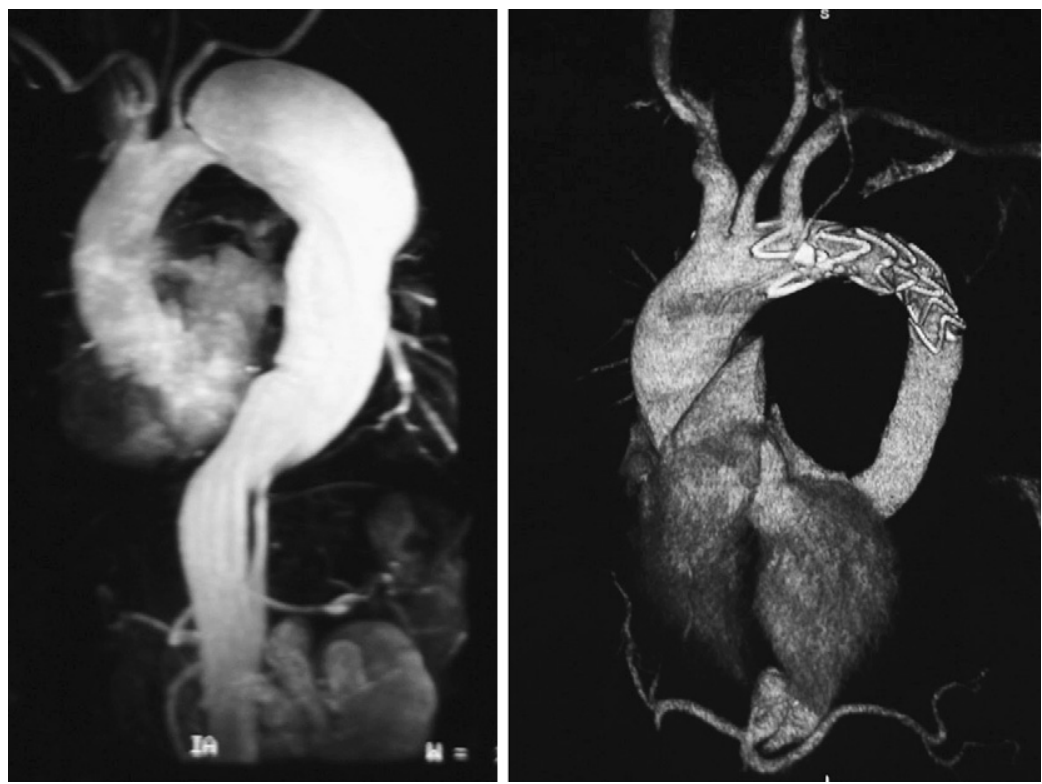

FIGURE 2. A, Magnetic resonance angiogram before thoracic endovascular aortic repair. B, Computed tomographic angiogram after thoracic endovascular aortic repair. 
TABLE 2. Early results $(N=84)$

\begin{tabular}{lc}
\hline Mortality (no.) & $1(1.2 \%)$ \\
Incomplete seal (no.) & $7(8.3 \%)$ \\
Renal insufficiency (no.) & $2(4.5 \%)$ \\
Fever $\left(>38.0^{\circ} \mathrm{C}\right.$, no.) & $47(56.0 \%)$ \\
Back pain (no.) & $52(61.9 \%)$ \\
Operative time (h, mean $\pm \mathrm{SD})$ & $2.5 \pm 0.3$ \\
Blood loss (mL, mean $\pm \mathrm{SD})$ & $86 \pm 6.5$ \\
Hospital stay (d, mean $\pm \mathrm{SD})$ & $10 \pm 2.0$ \\
\hline
\end{tabular}

medical treatment of uncomplicated dissection and surgical treatment of complicated dissection; however, surgery carries high morbidity and mortality. ${ }^{7}$ Good long-term outcome of medical treatment has been reported by some authors ${ }^{8}$; however, the results of other studies have not been so favorable. Mortality among patients who survive to hospital discharge with acute type B aortic dissection is high, approaching 1 in every 4 patients at 3 years. ${ }^{9}$ More and more evidence has shown a patent false lumen to be an independent risk factor for aortic enlargement and rupture during follow-up. Continuing enlargement of aorta has been noted during the chronic phase. ${ }^{10,11}$ Some authors have pointed out that if the maximum aortic diameter is less than $45 \mathrm{~mm}$ at the onset of the dissection, the chance of later enlargement is small ${ }^{12}$; others, however, have argued that small aortic diameter $(<40 \mathrm{~mm})$ does not lessen the chance of future enlargement. ${ }^{10}$ Thus, although it is not a current practice in all centers, we have made it a policy to treat all patients with chronic type B dissection with a patent false lumen. Our experience with TEVAR for acute type B dissection showed a 4-year survival of $89.4 \%$. A 5-year survival of $84.4 \%$ with TEVAR for chronic type B dissection is reported in this study. Both survivals are better than with traditional therapy. TEVAR for treatment of Type B dissection is still in its infancy, however, and many factors remain unresolved, such as the proper indication, anatomic requirement, choice of stent graft, and anesthesia.

Aortic dissections are divided into 3 phases in our center: acute ( $<2$ weeks), subacute ( 2 weeks -1 month), and chronic (>1 month). A 1-month interval was used to discriminate chronic dissection from acute dissection. After 1 month, both the adventitia and dissected intima, which are fragile in the acute phase, become fibrotic. Kato and coworkers ${ }^{4}$ share this opinion with us.

One patient with a huge false lumen aneurysm $(6 \mathrm{~cm}$ in diameter) and a large reentry in the abdominal aorta near the diaphragm died suddenly on postoperative day 1 . Sudden rupture of the descending thoracic aorta was confirmed by thoracentesis. The primary entry tear near the LSCA was sealed completely during TEVAR. The reentry in the abdominal aorta was left untreated. Before TEVAR, the false lumen had an entry and a reentry. After TEVAR, the reentry became an entry, and there was no longer any outlet. The blood pressure in the false lumen therefore increased. ${ }^{13}$ The elevation of the blood pressure in the false lumen was considered the cause of the rupture. The window
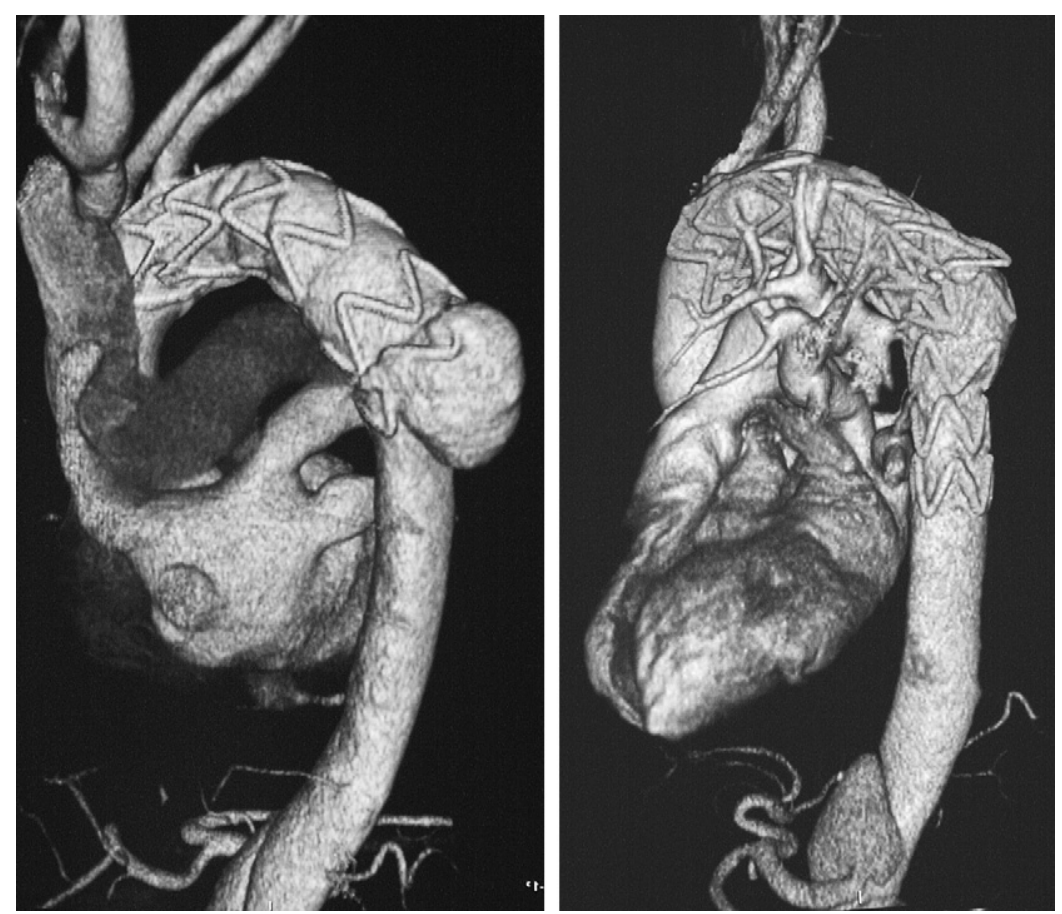

FIGURE 3. Patient with new intimal tear during follow-up. A, New intimal tear caused by distal end of stent graft after first thoracic endovascular aortic repair. B, Second stent graft was added at distal end of first one. 


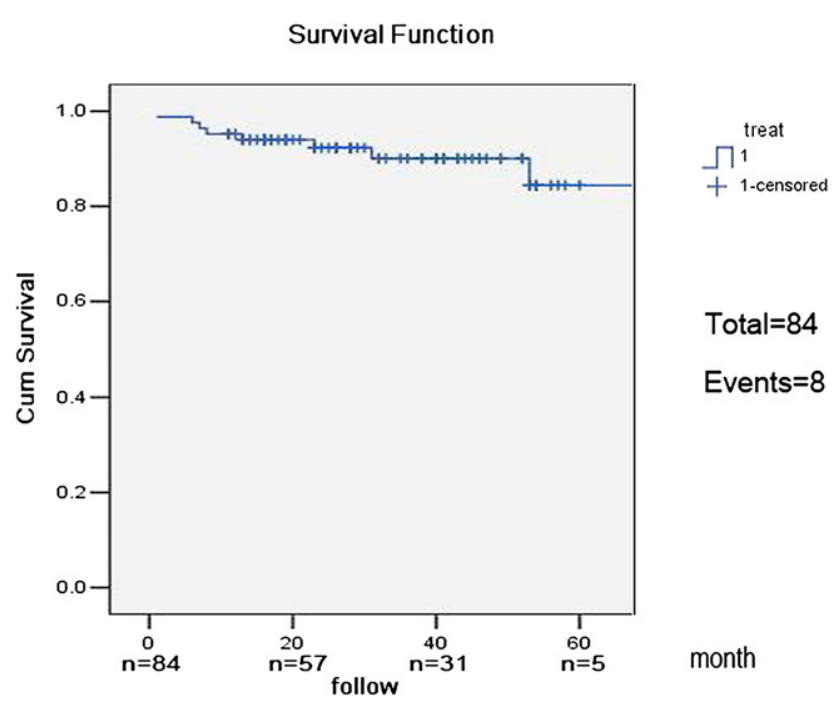

FIGURE 4. Kaplan-Meier survival curve shows 5-year survival of $84.4 \%$.

between sealing of the entry tear and thrombosis of the false lumen is a dangerous period that may last from minutes or hours to several weeks. High blood pressure, a large false lumen, a large reentry, and a short distance between the entry and reentry are considered risk factors for false lumen rupture during this window phase. After our experience with this patient, patients were sedated for at least 24 hours after TEVAR. Blood pressure was strictly controlled. Any reentry in the descending thoracic aorta was sealed by adding a cuff or cuffs (short stent graft) during TEVAR. Fortunately, with these measures, no more sudden deaths within a short time after TEVAR occurred.

Seven of 84 patients in our group had incomplete seal of the entry tear. The incidence of endoleak was $8.3 \%$, which was similar to that in Kim and colleagues' study. ${ }^{14}$ Partial thrombosis of the false lumen was observed in all 7 of the patients with incomplete seals. Three of these patients underwent a second TEVAR procedure. At first, partial thrombosis of the false lumen was considered to be better than a patent false lumen. That 3 of 4 patients without any further treatment would eventual die of rupture of the descending thoracic aorta was not anticipated. The cause of the rupture may have been that partial thrombosis of the false lumen led to the occlusion of the distal tear. The outflow of the false lumen was impeded or totally occluded, resulting in elevation of the blood pressure in the false lumen and eventually rupture of the false lumen. Tsai and coworkers ${ }^{15}$ concluded from the International Registry of Acute Aortic Dissection study that patients with a partially thrombosed false lumen had a worse prognosis than did those with a patent false lumen. This is similar to what we found in our study.

Because the prognosis of the endoleak is poor, all efforts to avoid it should be made. The shorter the distance between the opening of the LSCA and the entry tear, the greater the chance of an endoleak. In the later period of this study,
Freedom from Death, Endoleak or Reintervention

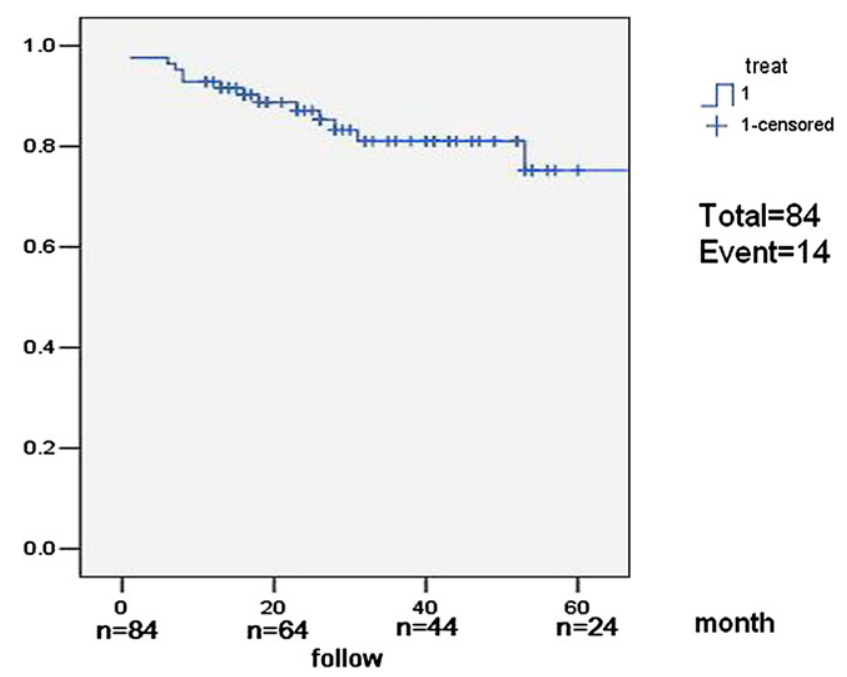

FIGURE 5. Kaplan-Meier curve shows freedom from death, endoleak, and reintervention at 7 years of $75.2 \%$.

another inclusion criterion for TEVAR was added, that the distance between the entry tear and the opening of the LSCA be no less than $10 \mathrm{~mm}$. From then on, endoleak was avoided. For those patients in whom the entry tear is closer to the LSCA, surgical treatment is the recommended option.

One patient with Marfan syndrome died of rupture of the abdominal aorta caused by continuous dilation of the abdominal aorta. Since then, TEVAR has no longer been used for patients with Marfan syndrome or any connective tissue diseases. Replacement of the diseased aorta with a vascular graft is the choice for these patients.

One patient had retrograde type A aortic dissection 1 month after TEVAR. This serious complication of TEVAR has been reported previously. ${ }^{16,17}$ The causes of type A dissection have been discussed in our previous article. ${ }^{18}$ The new intimal tear was located at the proximal end of the stent graft and may have been caused by the bare spring. The more rigid the stent graft, the greater the chance of a new intimal tear. Now flexible stent grafts (without a longitude strut) are used for TEVAR for aortic dissection in our center. When the whole piece is flexible, the proximal bare spring becomes less harmful.

In TEVAR for aortic dissection, the size of the stent graft is determined by the diameter of the proximal landing zone (usually the aortic arch). After deployment, the proximal portion of the stent graft anchors in the intact aortic arch, and the distal portion anchors in the true lumen of the dissected descending aorta. In the early period of the study, we did not have any domestic stent grafts. Talent and EndoFit were our only choices for TEVAR; however, both of these were designed for true aneurysm rather than 
dissection. They did not have a tapered design. During follow-up, some patients returned with a significant dilated descending aorta caused by dilatation of the distal part of the stent graft. One patient even had a new intimal tear caused by dilatation of the distal end of the stent graft (Figure 3). An aneurysm or a new tear at the end of the stent graft has been previously reported by others. ${ }^{17,19} \mathrm{We}$ suspected that there might have been a significant difference in diameter between the arch and the true lumen in the descending aorta. The taper ratio between the arch and the true lumen in the descending aorta has been studied. ${ }^{20}$ The taper ratio is defined as [(Arch $\Phi$ - True lumen $\Phi) /$ Arch $\Phi] \cdot 100 \%$ ( $\Phi$ : diameter). The study showed a taper ratio of $30 \%$ in chronic dissection. If a stent graft without a tapered design and $15 \%$ larger than the aortic arch is selected, its distal end will exceed the true lumen of the descending aorta by more than $60 \%$. This would explain the excessive dilatation of the descending aorta and the new intimal tears during follow-up.

Domestic stent grafts were available later in the study. Since then, a tapered stent graft (proximal end $6 \mathrm{~mm}$ larger than distal end) has been used in TEVAR for aortic dissection, and none of patients so treated had any obvious dilatation of the descending aorta during follow-up.

Effective analgesia and steady blood pressure control are keys to the success of the procedure. In the beginning, general anesthesia was used. A smooth process could be achieved, and the blood pressure was easy to control. Then a patient with a pseudoaneurysm had a cerebral infarction during TEVAR, and we were unable to determine when it occurred. We realized that the patient's conscious state should be monitored during the process, and we switched to epidural and local anesthesia. When epidural anesthesia was used, some anesthesiologists were concerned about epidural hematoma. Finally, local anesthesia was chosen during TEVAR. Apart from the conscious state, another benefit was found to this in that the spinal cord function could also be monitored during the procedure.

The limitations of this study are obvious. It is a retrospective, uncontrolled study. During the study, the inclusion criteria were changed. Some stent grafts were used in the early period of the study but abandoned later. Although the longest follow-up is 7 years, the mean follow-up is not quite 3 years. All these limitations are explained in part by the newness and fast development of this novel technique. Further investigations are required in the future, but we are optimistic about TEVAR. We believe that, with refinement of the stent graft and increased surgical experience, TEVAR has the potential to become the first choice for aortic dissection.

\section{CONCLUSIONS}

Early and midterm results showed TEVAR to be effective for the treatment of chronic type B aortic dissection.
Endoleak was the main cause of death during follow-up. A flexible and tapered stent graft may be more suitable for aortic dissection. With increased surgical experience and refinement of the stent graft, better outcomes are expected.

\section{References}

1. Volodos NL, Karpovich IP, Troyan VI, Kalashnikova YuV, Shekhanin VE, Ternyuk NE, et al. Clinical experience of the use of self-fixing synthetic prostheses for remote endoprosthetics of the thoracic and the abdominal aorta and iliac arteries through the femoral artery and as intraoperative endoprosthesis for aorta reconstruction. Vasa Suppl. 1991;33:93-5.

2. Dake MD, Miller DC, Semba CP, Mitchell RS, Walker PJ, Liddell RP. Transluminal placement of endovascular stent-grafts for the treatment of descending thoracic aortic aneurysms. N Engl J Med. 1994;331:1729-34.

3. Nienaber CA, Fattori R, Lund G, Dieckmann C, Wolf W, von Kodolitsch Y, et al Nonsurgical reconstruction of thoracic aortic dissection by stent-graft placement N Engl J Med. 1999;340:1539-45.

4. Kato N, Shimono T, Hirano T, Suzuki T, Ishida M, Sakuma H, et al. Midterm results of stent-graft repair of acute and chronic aortic dissection with descending tear: the complication-specific approach. J Thorac Cardiovasc Surg. 2002;124: 306-12.

5. Eggebrecht $H$, Nienaber $C A$, Neuhäuser $M$, Baumgart $D$, Kische $S$, Schmermund A, et al. Endovascular stent-graft placement in aortic dissection a meta-analysis. Eur Heart J. 2006;27:489-98.

6. Xu SD, Huang FJ, Yang JF, Li ZZ, Wang XY, Zhang ZG, et al. Endovascular repair of acute type B aortic dissection: early and mid-term results. J Vasc Surg. 2006;43:1090-5.

7. Dudra J, Shiiya N, Matsui Y, Sakuma M, Ishii K, Asada M, et al. Operative results of thoracoabdominal repair for chronic type B aortic dissection. J Cardiovasc Surg (Torino). 1997;38:147-51.

8. Hata M, Shiono M, Inoue T, Sezai A, Niino T, Negishi N, et al. Optimal treatmen of type B acute aortic dissection: long-term medical follow-up results. Ann Thorac Surg. 2003;75:1781-4.

9. Tsai TT, Fattori R, Trimarchi S, Isselbacher E, Myrmel T, Evangelista A, et al. Long-term survival in patients presenting with type B acute aortic dissection: insights from the International Registry of Acute Aortic Dissection. Circulation. 2006;114:2226-31.

10. Sueyoshi E, Sakamoto I, Hayashi K, Yamaguchi T, Imada T. Growth rate of aortic diameter in patients with type B aortic dissection during the chronic phase. $\mathrm{Circu}$ lation. 2004;110(11 Suppl. 1):II256-61.

11. Blount KJ, Hagspiel KD. Aortic diameter, true lumen, and false lumen growth rates in chronic type B aortic dissection. AJR Am J Roentgenol. 2009;192: W222-9.

12. Kunishige H, Myojin K, Ishibashi Y, Ishii K, Kawasaki M, Oka J. Predictors of surgical indications for acute type $\mathrm{B}$ aortic dissection based on enlargement of aortic diameter during the chronic phase. Jpn J Thorac Cardiovasc Surg. 2006;54: 477-82.

13. Tsai TT, Schlicht MS, Khanafer K, Bull JL, Valassis DT, Williams DM, et al. Tear size and location impacts false lumen pressure in an ex vivo model of chronic type B aortic dissection. $J$ Vasc Surg. 2008;47:844-51.

14. Kim U, Hong SJ, Kim J, Kim JS, Ko YG, Choi D, et al. Intermediate to long-term outcomes of endoluminal stent-graft repair in patients with chronic type B aortic dissection. J Endovasc Ther. 2009;16:42-7.

15. Tsai TT, Evangelista A, Nienaber CA, Myrmel T, Meinhardt G, Cooper JV, et al Partial thrombosis of the false lumen in patients with acute type B aortic dissection. N Engl J Med. 2007;357:349-59.

16. Neuhauser B, Greiner A, Jaschke W, Chemelli A, Fraedrich G. Serious complications following endovascular thoracic aortic stent-graft repair for type B dissection. Eur J Cardiothorac Surg. 2008;33:58-63.

17. Dong ZH, Fu WG, Wang YQ, Guo da Q, Xu X, Ji Y, et al. Retrograde type A aortic dissection after endovascular stent graft placement for treatment of type B dissection. Circulation. 2009;119:735-41.

18. Xu SD, Li ZZ, Huang FJ, Yang JF, Wang XY, Zhang ZG, et al. Treating aortic dissection and penetrating aortic ulcer with stent graft: thirty cases. Ann Thorac Surg. 2005;80:864-8.

19. Lopera J, Patino JH, Urbina C, García G, Alvarez LG, Upegui L, et al. Endovascular treatment of complicated type-B aortic dissection with stent-grafts: midterm results. J Vasc Interv Radiol. 2003;14:195-203.

20. Xu SD, Huang FJ, Du JH, Li Y, Fan ZM, Yang JF, et al. A study of aortic dimension in type B aortic dissection. Interact Cardiovasc Thorac Surg. 2008;7:244-8. 